December 14, 2020

\title{
Exploring the Great Pyramid: Stand-Alone Monte Carlo Simulations for Detector Optimization
}

\author{
Tabitha Welch, University of Chicago
}

\begin{abstract}
Cosmic-ray muon imaging has been used to non-destructively examine the Pyramids of Khufu and Khafre on the Giza Plateau; the EGP project will continue this line of research by undertaking a full tomographic scan of the former and, in doing so, will increase the sensitivity of the technique by upwards of two orders of magnitude. This paper details the development of stand-alone Monte Carlo simulations intended to aid in optimizing the EGP detector system. While the rectangular scintillator model considered here does provide sufficient position resolution in a few specific test cases, it is not the optimal telescope design. An attempted detector modification to permit tagging of muons by their incident momenta is also discussed.
\end{abstract}

\section{Contents}

1 Introduction $\quad 2$

1.1 Historical Background . . . . . . . . . . . . . . . . . . 2

1.2 Motivation . . . . . . . . . . . . . . . . . . . 2

2 EGP Detector Proposal 2

3 Coordinate System $\quad 4$

4 Simulation Parameters 6

4.1 Removing Tracking of Secondary Particles to Improve Simulation Speed . . . 8

5 Multiplicity $\quad \mathbf{8}$

5.1 Counter Response . . . . . . . . . . . . . . . . . . . 10

6 Positional Back-projection and Comparison with Concrete Scattering 12

6.1 Example Study: Void 25 m from Pyramid Surface . . . . . . . . . . . . . . . 14

7 Use of $\frac{d E}{d x}$ in Determining Muon Momentum $\quad 15$

7.1 Basic Detector Design vs. Additional Pb Layer . . . . . . . . . . . . . . . 16

8 Summary $\quad 17$

9 Acknowledgments $\quad 18$

This manuscript has been authored by Fermi Research Alliance, LLC under Contract No. DE-AC02-07CH11359 with the U.S. Department of Energy, Office of Science, Office of High Energy Physics. 


\section{Introduction}

Commissioned in the 26th century BC by Khufu, the second of ancient Egypt's Fourth Dynasty pharaohs, the Great Pyramid at Giza continues to inspire excitement and spark debate today. There is no universal consensus as to how the pyramid was built or why its internal complexity so far exceeds the apparent complexity of subsequent pyramids. Because undertaking any kind of intrusive excavation would have serious ramifications for Egypt, both culturally and economically, these mysteries have been difficult to solve. However, a noninvasive scanning method pioneered by Alvarez et al. may provide a unique solution to this dilemma. This technique relies upon cosmic-ray muon tomography and makes use of modern high-energy physics detector technology. The Exploring the Great Pyramid mission (EGP), a collaboration between Fermilab, the University of Chicago, the University of Virginia, and Yale, will undertake a new exploration of Khufu's Pyramid with the aid of these tools. This paper describes the development of a stand-alone Monte Carlo simulation intended to help optimize the proposed EGP detector system.

\subsection{Historical Background}

In 1970, L. Alvarez et al. published an article in Science that detailed their scan of Khafre's pyramid using cosmic-ray muon radiography [1]. This experimental setup involved placing two 1.8-meter square spark chambers in Khafre's Belzoni Chamber, which lies near the center of the pyramid's base. Alvarez' main goal was to locate previously undiscovered chambers; this was driven by the apparently stark differences in internal complexity between this pyramid and the Great Pyramid, its predecessor. While the study found no such chambers, the technique itself was shown to be successful.

Alvarez' exploration was followed in 2017 by the ScanPyramids collaboration, whose study of the Great Pyramid identified a previously unknown void above the Grand Gallery [2]. This void, which is a minimum of $30 \mathrm{~m}$ in length and has a cross-section like that of the Grand Gallery, is the first major structure to be discovered within the pyramid since the 1800s. The ScanPyramids group also used muon radiography as their primary tool.

\subsection{Motivation}

Although the endeavors described above have been very successful, numerous open questions remain regarding the Great Pyramid's interior. The EGP project, unlike past experiments, will apply true tomographic imaging and reconstruction techniques; this will allow us to distinguish not only voids from solid stone, but also much subtler variations in density. The proposed EGP detectors also possess about 100x the surface area of the ScanPyramids detectors, resulting in significantly higher resolution. Equipped with these tools, we may be able to shed light on the nature of the ScanPyramids void, discover additional clues as to the pyramid's construction, and perhaps even uncover additional unknown unknowns within the structure.

\section{EGP Detector Proposal}

Figure 1 depicts the proposed EGP telescope design. The detectors will be housed in 40-ft climate-controlled shipping containers, rendering them extremely portable and durable; 
there will be banks of scintillator strips inside, read out by silicon photomultipliers and separated by a distance of $2 \mathrm{~m}$. Each bank will consist of two side-by-side arrays, as shown, and each array will be composed of three horizontal and six vertical modules, yielding a total of 12 horizontal modules and 24 vertical modules per container. Individual horizontal scintillator strips will measure $2 \mathrm{~cm} \mathrm{x} 2 \mathrm{~cm} \mathrm{x} 4.8 \mathrm{~m}$, while vertical strips will measure $2 \mathrm{~cm}$ x $2 \mathrm{~cm} \times 2.4 \mathrm{~m}$. These cargo containers will be stacked in $2 \times 2$ arrays, for a total surface area of $92.16 \mathrm{~m}^{2}$ per detector unit.

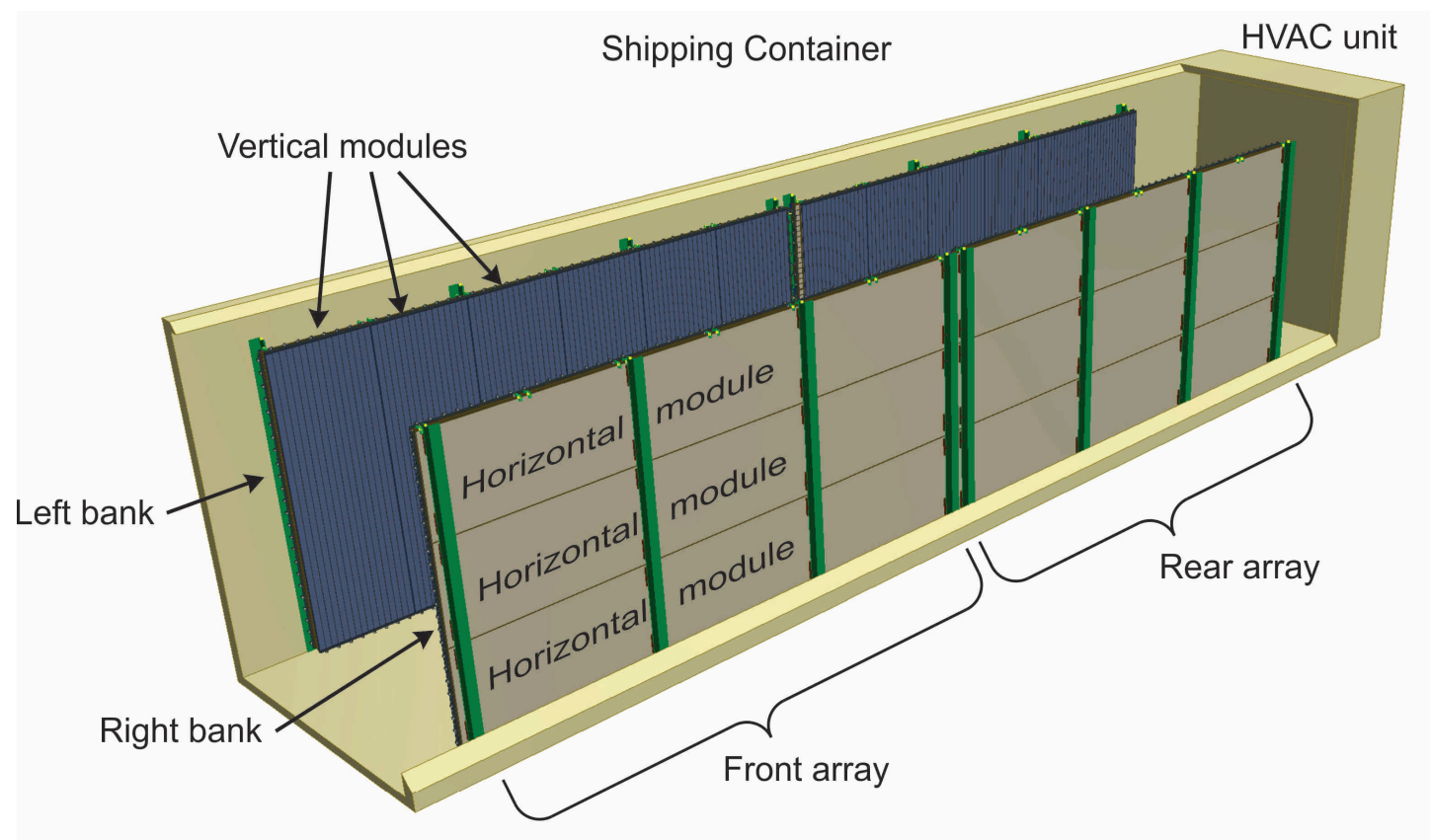

Figure 1: Diagram of detectors in a temperature-controlled cargo container. Each array of detectors will consist of three horizontal and six vertical modules, and there will be two arrays per bank, for a total of 12 horizontal modules and 24 vertical modules in a given container.

The telescopes will be placed around the pyramid at approximately $25 \mathrm{~m}$ from its base and moved along the perimeter periodically during the anticipated 1-2 year viewing time. Figure 2 shows a few candidate detector locations, which are somewhat restricted by the presence of other ancient and modern structures around the pyramid. The north and west sides are most accessible. 


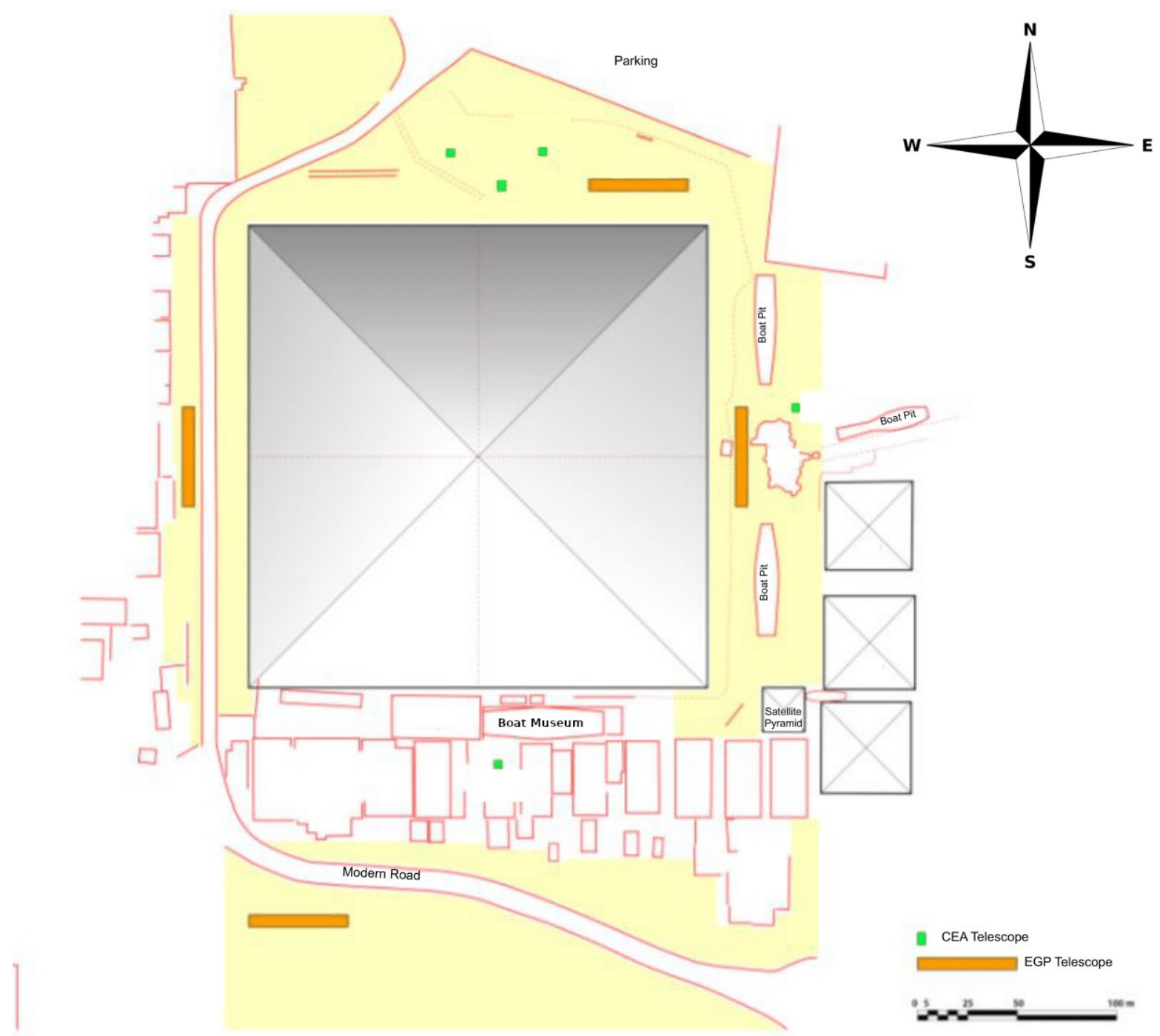

Figure 2: Plan view of the pyramid and its immediate surroundings, with a few potential detector placements marked in orange. The north and west sides are the most accessible, while the east and south sides pose a greater challenge due to surrounding structures like the boat museum.

\section{Coordinate System}

The coordinate system used in all of the following simulations is shown in Figures 3, 4, and 5. Figures 3 and 4 include plan and elevation views of the pyramid, with a simplified proposed detector setup for reference. We make use of both global and local coordinates; the global Cartesian coordinates are designated $(\mathrm{X}, \mathrm{Y}, \mathrm{Z})$, and their polar counterparts, used for describing muon trajectories, are $(\theta, \phi)$. The global coordinates' origin is located at the center of the pyramid's base, and the $\mathrm{X}$ axis points east, while the $\mathrm{Y}$ axis points north and the $\mathrm{Z}$ axis points upwards. The local Cartesian coordinates we use are $(\mathrm{u}, \mathrm{v})$; they span a given detector plane, with the origin at the bottom left corner of that plane from the perspective facing the pyramid. 


\section{Elevation View - Container on West Face}

Not to scale
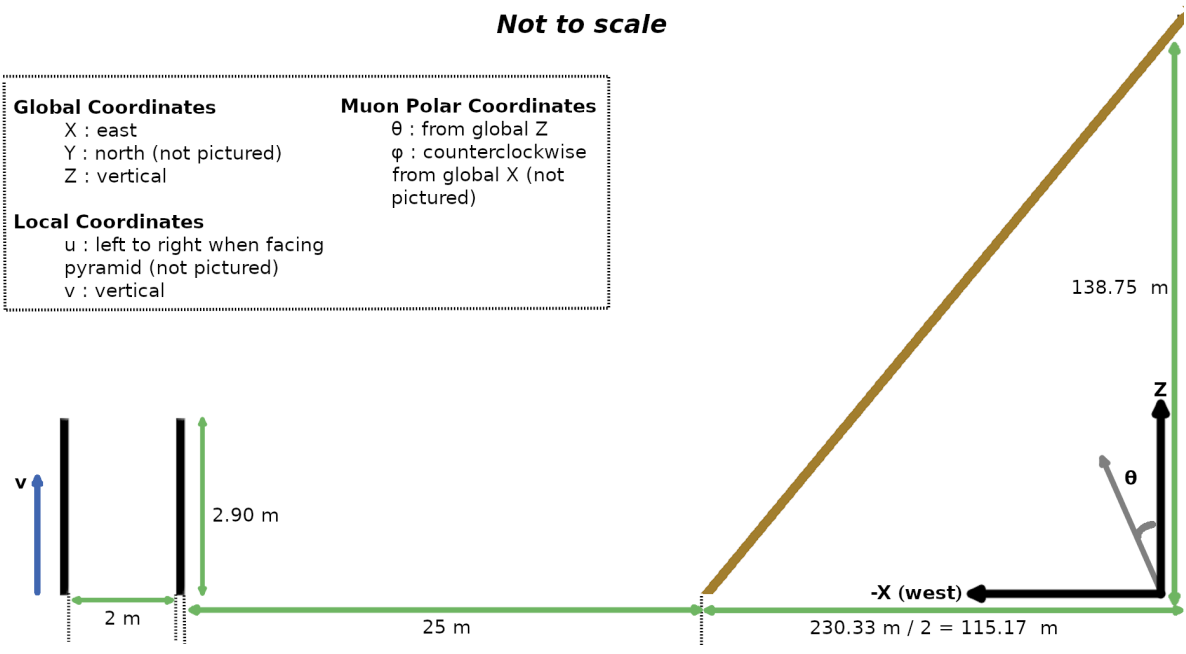

Figure 3: Elevation view of coordinate system used for all simulations, with the pyramid for reference. We define both local coordinates $(\mathrm{u}, \mathrm{v})$ - to describe locations on a given detector plane - and global coordinates $(\mathrm{X}, \mathrm{Y}, \mathrm{Z})$, as well as polar coordinates $(\theta, \phi)$ to describe muon trajectories. The origin of the global coordinates is at the center of the pyramid's base, while the origin of the local coordinates is at the corner of the relevant detector plane (see Fig. 5).

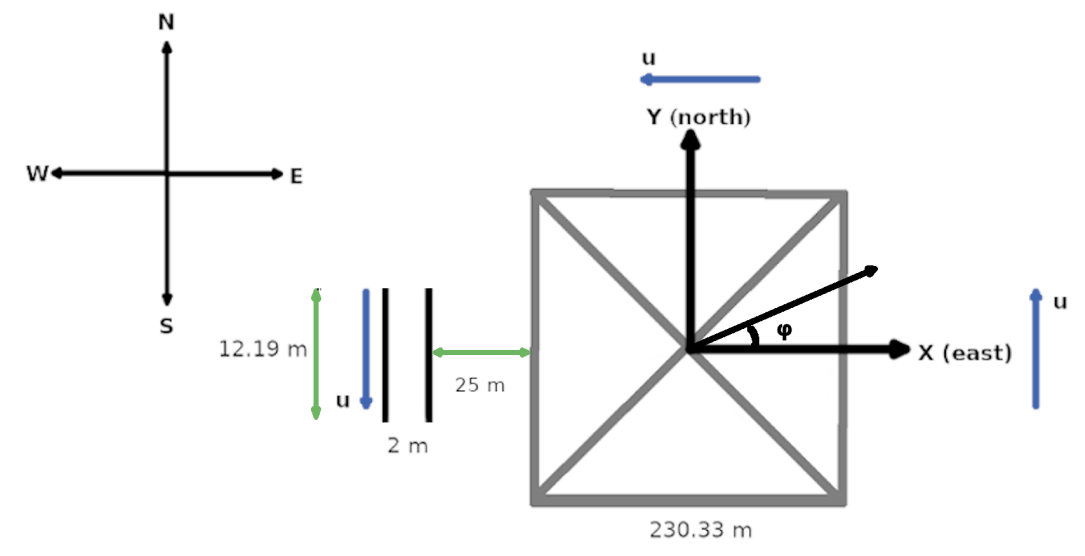

Plan View - Container on West Face Not to scale

(Please see elevation view for a complete list of relevant coordinates)

Figure 4: Plan view of the coordinate system. The global $\mathrm{X}$ axis points east, while the global $\mathrm{Y}$ axis points north; $\mathrm{Z}$ points upwards out of the page. The orientation of the local $\mathrm{u}$ axis varies by specific container placement, as shown, and the local $\mathrm{v}$ axis always points up. 

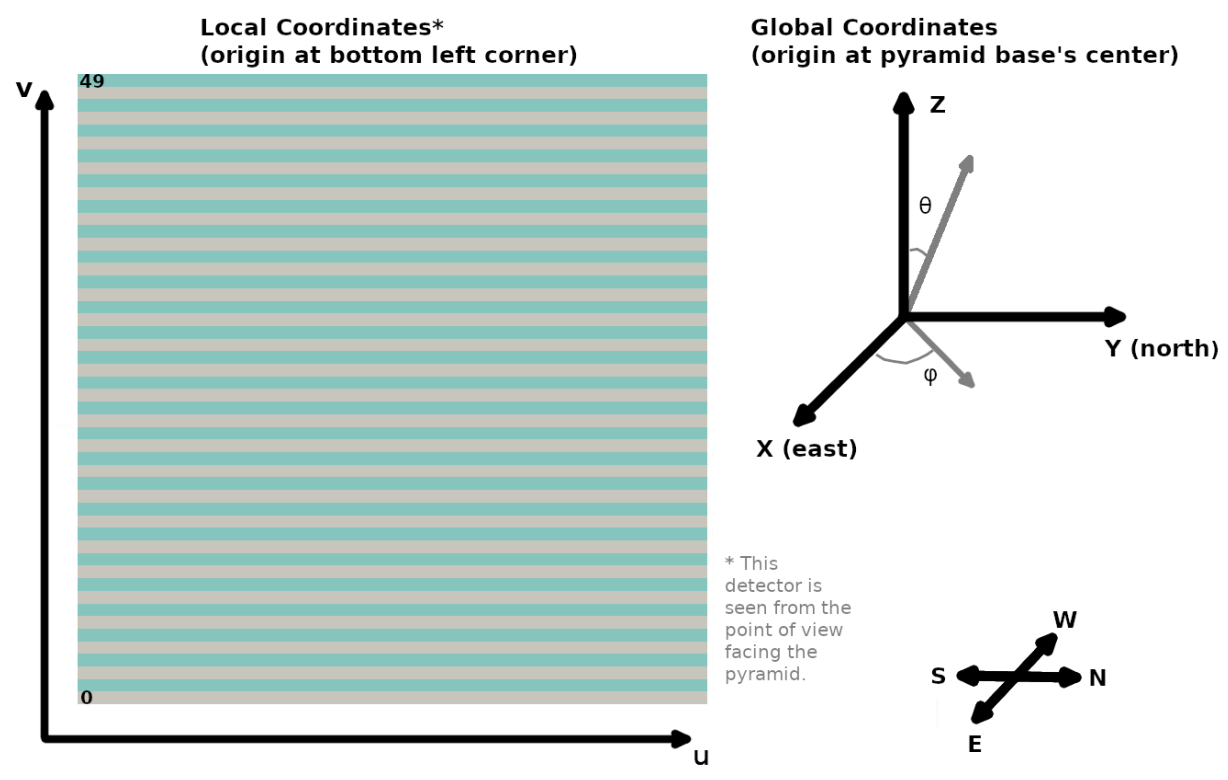

Figure 5: Detailed coordinate system.

\section{Simulation Parameters}

We developed a toy model of the proposed detectors based on a rectangular scintillator design. Simulations were carried out with the GEANT4 toolkit [4, 5]; supporting analysis was performed in Root [6,7], and all detector visualizations were produced with Paraview [8]. The primary objectives of this study were to determine the positional resolution needed in light of multiple scattering effects and to investigate the possibility of tagging muons by their incident momenta.

As shown in Figure 6, the toy model consists of two $1 \mathrm{~m} \times 1 \mathrm{~m}$ detectors. These correspond to the detectors on the two walls of the cargo container discussed above, so there is a $2 \mathrm{~m}$ gap between them. Each detector is composed of two planes of 50 rectangular scintillator strips, and each strip has dimensions of $2 \mathrm{~cm} \mathrm{x} 2 \mathrm{~cm} \times 1 \mathrm{~m}$. In each detector, one set of strips is vertical and the other is horizontal, which yields a set of $\mathrm{u}$ and $\mathrm{v}$ coordinates for every hit (see section 6 for details). These detectors sit in a vacuum; no container walls, electronics, or fibers are included in the simulation.

G4ParticleGun, GEANT's simple built-in particle gun, serves as the muon source for the model. This gun produces one muon at a time, with exactly the same initial momentum and position each time. In a given run of the simulation, a pre-determined number of these identical muons are fired at the detectors, and information about their energy deposition in the scintillator strips is recorded (see below). 


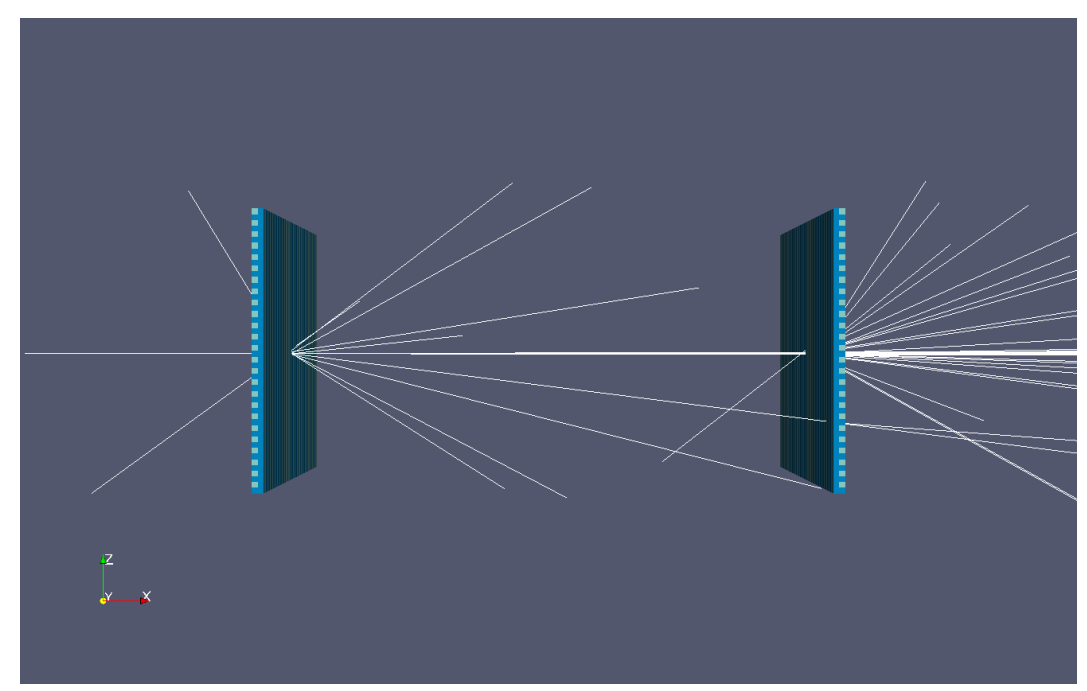

Figure 6: The simulation contains two detectors, each consisting of two $1 \mathrm{~m} \mathrm{x} 1 \mathrm{~m}$ scintillator planes and each plane composed of $502 \mathrm{~cm} \mathrm{x} 2 \mathrm{~cm} \times 1 \mathrm{~m}$ strips; there is a $2 \mathrm{~m}$ gap between the first and second detectors. In the above visualization, tracks from $1004 \mathrm{GeV}$ muons and all of their associated secondaries are shown, each muon beginning to the left of detector 1 . (Note: the secondary particle removal discussed in section 4.1 applies only to the concrete volume and is therefore not relevant here.)

For studies involving multiple-scattering effects from the pyramid itself, we added a concrete block $25 \mathrm{~m}$ upstream of the detectors to simulate the pyramid. This is shown in Figure 7. We varied the thickness of this block in order to represent potential voids at different depths inside the pyramid; a 115-m block corresponded to a hypothetical chamber at the pyramid's center and was therefore the greatest thickness of interest (see Fig. 3).

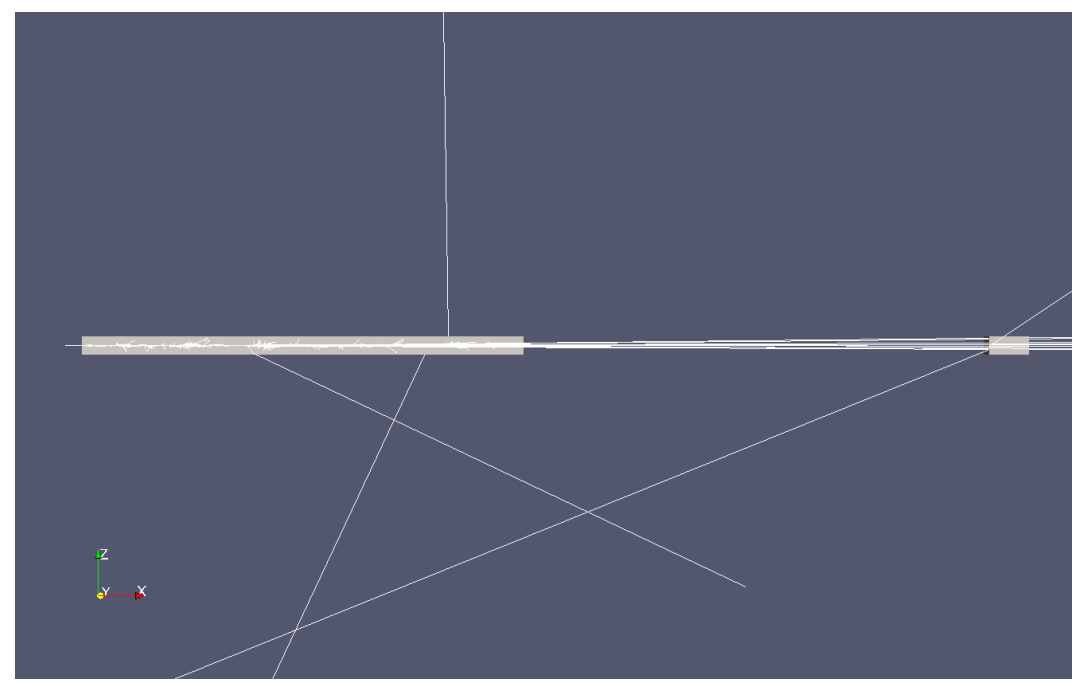

Figure 7: A concrete block that served as a pyramid dummy was later added $25 \mathrm{~m}$ upstream of the detectors. The width and height of this block matched that of the detectors; its length was varied between 1 and $115 \mathrm{~m}$. In this visualization, the block is $25 \mathrm{~m}$ long and is shown with tracks from $1050 \mathrm{GeV}$ muons and their secondaries. No secondaries that were generated in the concrete with energy greater than $10 \mathrm{MeV}$ are shown (see section 4.1 for details). The detector is shown in gray at the right side of the image. 


\subsection{Removing Tracking of Secondary Particles to Improve Simu- lation Speed}

Particularly with the addition of the concrete block discussed above, simulation speed became a major concern. Since most secondary particles produced inside the concrete have no impact whatsoever on the results, it is completely unnecessary to track them for our purposes. So, a cut was implemented within the concrete volume that resulted in all secondaries below the threshold energy being killed immediately upon creation. Table 1 details the timing results from these changes. For most runs with concrete, a 10-MeV cutoff was used.

\begin{tabular}{|c|c|c|c|}
\hline Concrete Thickness (m) & All Secondaries & $\begin{array}{l}10 \mathrm{MeV} \text { Sec- } \\
\text { ondary Cutoff in } \\
\text { Concrete }\end{array}$ & $\begin{array}{l}10 \mathrm{GeV} \text { Secondary } \\
\text { Cutoff in Concrete }\end{array}$ \\
\hline 50 & $\begin{array}{l}\text { User }=408 \mathrm{~s} \\
\text { Real }=1830 \mathrm{~s} \\
\text { Sys }=322 \mathrm{~s}\end{array}$ & $\begin{array}{l}\text { User }=93.1 \mathrm{~s} \\
\text { Real }=424 \mathrm{~s} \\
\text { Sys }=76.8 \mathrm{~s}\end{array}$ & $\begin{array}{l}\text { User }=36.7 \mathrm{~s} \\
\text { Real }=147 \mathrm{~s} \\
\text { Sys }=31.2 \mathrm{~s}\end{array}$ \\
\hline 25 & $\begin{array}{l}\text { User }=227 \mathrm{~s} \\
\text { Real }=954 \mathrm{~s} \\
\text { Sys }=178 \mathrm{~s}\end{array}$ & $\begin{array}{l}\text { User }=48.0 \mathrm{~s} \\
\text { Real }=199 \mathrm{~s} \\
\text { Sys }=40.6 \mathrm{~s}\end{array}$ & $\begin{array}{l}\text { User }=18.5 \mathrm{~s} \\
\text { Real }=72.6 \mathrm{~s} \\
\text { Sys }=15.9 \mathrm{~s}\end{array}$ \\
\hline 10 & $\begin{array}{l}\text { User }=86.2 \mathrm{~s} \\
\text { Real }=360 . \mathrm{s} \\
\text { Sys }=67.4 \mathrm{~s}\end{array}$ & $\begin{array}{l}\text { User }=19.0 \mathrm{~s} \\
\text { Real }=82.5 \mathrm{~s} \\
\text { Sys }=16.0 \mathrm{~s}\end{array}$ & $\begin{array}{l}\text { User }=7.54 \mathrm{~s} \\
\text { Real }=32.3 \mathrm{~s} \\
\text { Sys }=6.64 \mathrm{~s}\end{array}$ \\
\hline 5 & $\begin{array}{l}\text { User }=45.0 \mathrm{~s} \\
\text { Real }=183 \mathrm{~s} \\
\text { Sys }=35.5 \mathrm{~s}\end{array}$ & $\begin{array}{l}\text { User }=9.50 \mathrm{~s} \\
\text { Real }=42.5 \mathrm{~s} \\
\text { Sys }=7.98 \mathrm{~s}\end{array}$ & $\begin{array}{l}\text { User }=4.03 \mathrm{~s} \\
\text { Real }=15.9 \mathrm{~s} \\
\text { Sys }=3.35 \mathrm{~s}\end{array}$ \\
\hline
\end{tabular}

Table 1: Raw timing data from runs of 1,000 50-GeV muons with various concrete thicknesses. Introducing a $10-\mathrm{MeV}$ cut on secondaries generated inside the concrete resulted in a $4 \mathrm{x}$ speedup over keeping all secondaries, while a $10-\mathrm{GeV}$ cut, which essentially eliminated all concrete-generated secondaries, further increased the simulation's speed by about another factor of three. Note: in the above, "real" refers to the total time elapsed from the simulation's start to its finish (i.e., wall clock time). "User" and "sys" refer to CPU time spent outside and inside the Unix kernel, respectively; their sum represents the total CPU time.

\section{$5 \quad$ Multiplicity}

Appropriately reconstructing the position and angle of an incident muon depends heavily on the number of hits it generates in the detector planes. We therefore investigated the correlation between the hit multiplicity of a particle and its angle with respect to the detector. 

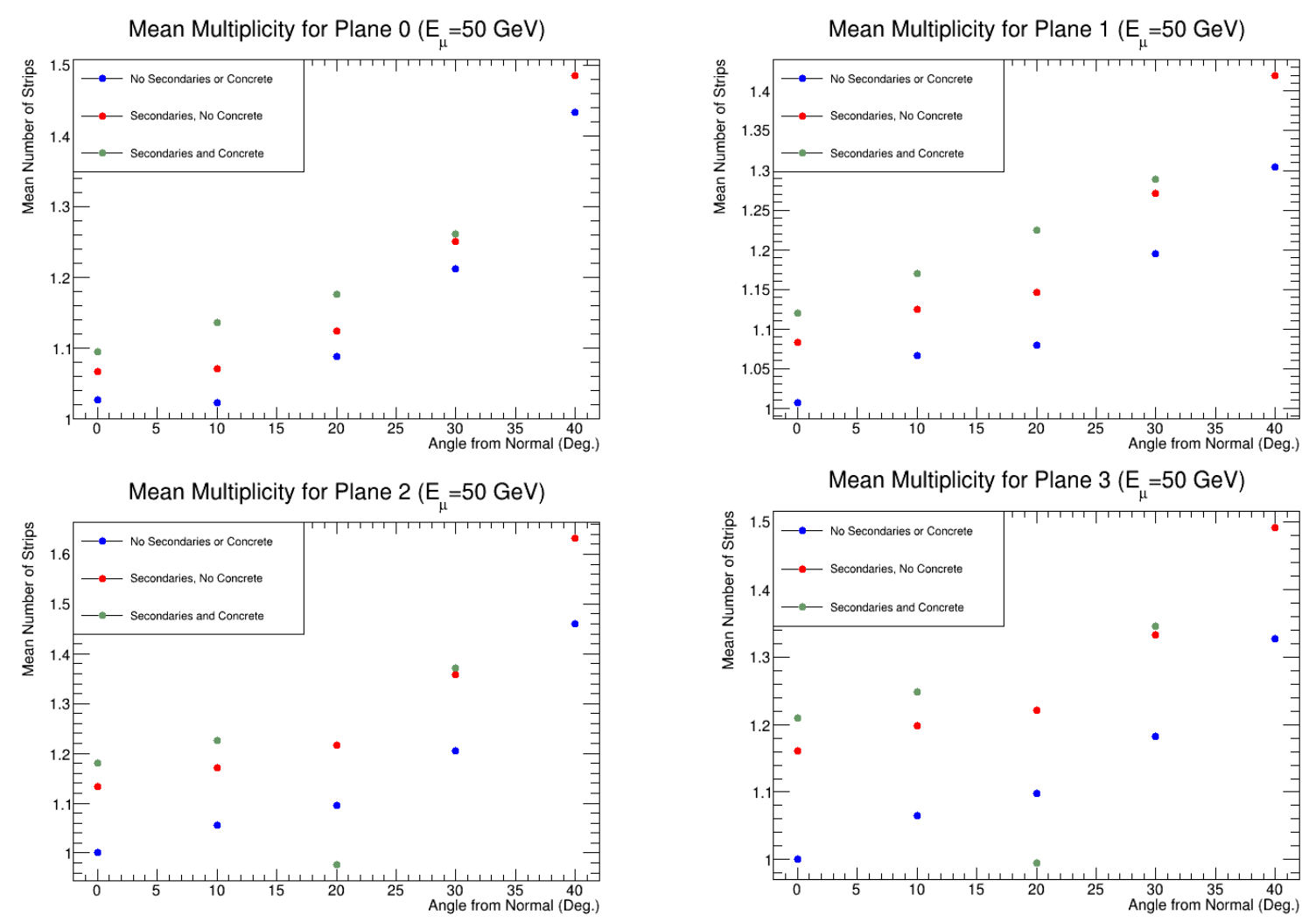

Figure 8: The mean hit multiplicity for each of the four detector planes, plotted as a function of incident angle. Each run consisted of 10,000 50-GeV muons, which were scanned across a full cell width $(2 \mathrm{~cm})$.

This analysis was repeated for three different cases: 1.) allowing no secondary particles (even from interactions in the scintillator) and including no concrete, 2.) allowing secondary particles, but including no concrete, and 3.) including both secondaries (up to the cut described in 4.1) and concrete. All data used an incident muon energy of $\mathrm{E}_{\mu}=50$ $\mathrm{GeV}$, and for this study only, the detector size was increased to $3 \mathrm{~m}$ x $3 \mathrm{~m}$ to accommodate the angles of interest. The size of the individual scintillator strips, as well as the 2-m gap between the first and second banks, remained unchanged. For the trials involving concrete, the block was positioned at the same angle as the muon beam - i.e., for every run, the particles passed through exactly $25 \mathrm{~m}$ of concrete, regardless of their angle with respect to the detectors. Unlike the other studies discussed here, the beam was scanned across a full cell width during each run.

Figure 8 shows the mean multiplicity in each individual plane for the three scenarios considered. All four planes show a consistent increase in multiplicity as incident angle increases. The trend is present even for runs with no secondary particles, which implies that it stems mainly from the geometry of the situation.

One simple approach to calculating hit position entails using only those events that result in a single hit per plane and rejecting the remainder. We refer to this as the "singles cut." Table 2 and Figure 9 depict the percentage of events that pass this cut in the three simulation scenarios discussed above. For angles close to normal, the majority of events survive; however, there is a sharp decrease in surviving events for wider angles. This is consistent with the multiplicity trends for individual planes (see above). So, while the singles cut is useful for near-normal incidence, it should not be applied to events with arbitrary 
angles. For the latter, a weighted mean or clustering algorithm is necessary. In section 6.1, only normal muons were used, and the singles cut was applied.

\begin{tabular}{|c|c|c|c|}
\hline Angle (Deg.) & No Secondaries & Secondaries, No Concrete & Secondaries and 25 m. Concrete \\
\hline 0 & 96.81 & 75.080 & 71.99 \\
\hline 10 & 90.73 & 68.55 & 62.87 \\
\hline 20 & 78.11 & 59.80 & 37.99 \\
\hline 30 & 65.54 & 49.12 & 29.56 \\
\hline 40 & 51.99 & 39.12 & - \\
\hline
\end{tabular}

Table 2: For each of the above three situations, the percentage of events that survive if we limit to only one hit per plane in all four planes. This is the "singles cut." Here, "angle" refers to the amount by which both $\theta$ and $\phi$ differ from normal incidence.

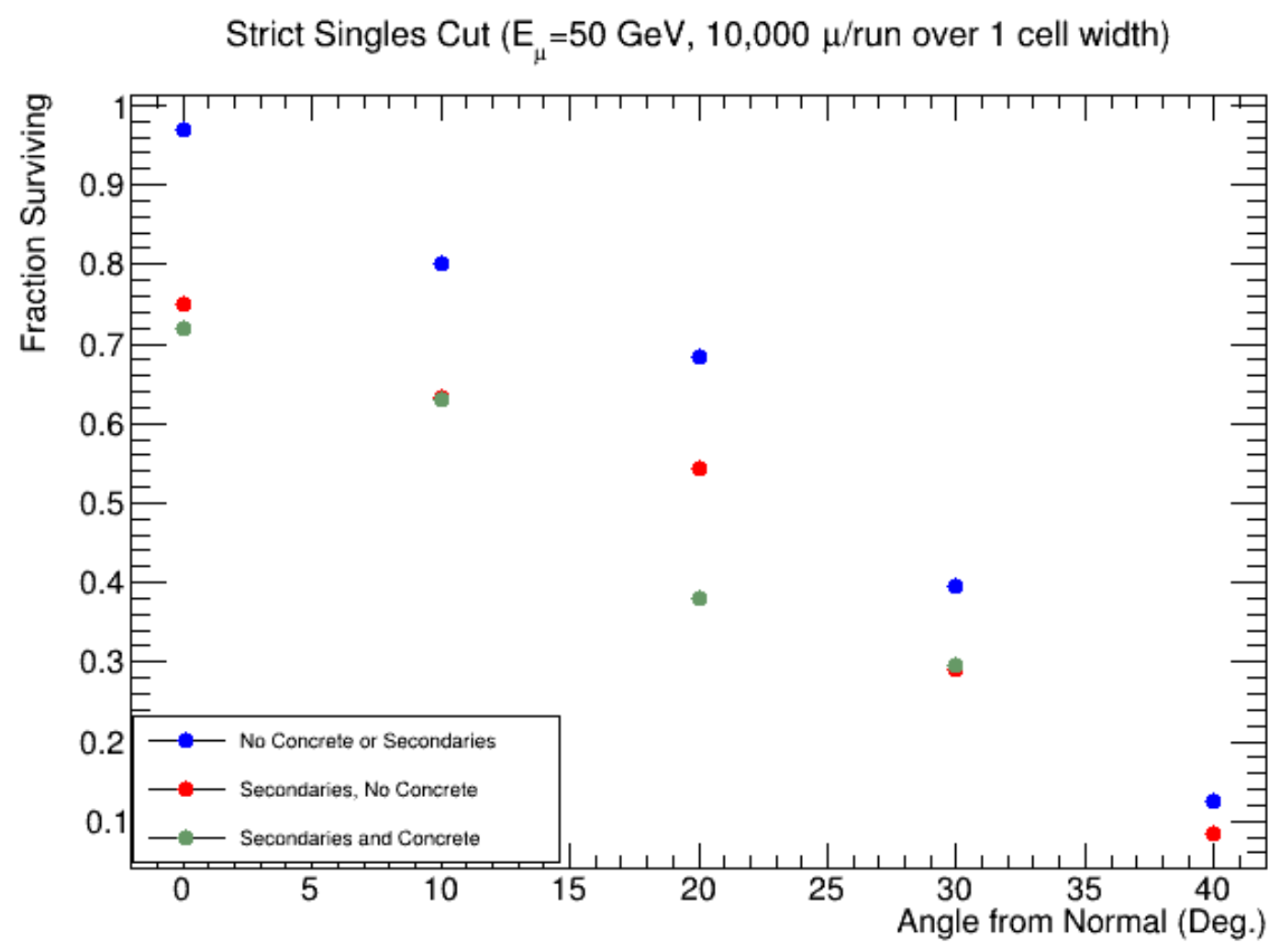

Figure 9: The fraction of events that pass the singles cut, plotted as a function of angle from normal incidence. The sharp decrease in surviving events as angle widens indicates that the singles cut is not appropriate for most muons of interest.

\subsection{Counter Response}

As the previous discussions imply, multiplicity is heavily dependent on the geometry of a given event in the detectors. To explore this pattern in greater detail, we calculated the expected response of a pair of adjacent counters, in terms of energy deposition, given a variety of incident angles and hit positions. The effects of secondary particles were disregarded; only the energy deposited by the primary muon was considered. 
A counter response map is shown in Figure 10. Data for this table were obtained by calculating the salient path length in two adjacent scintillator strips, then converting this quantity to $\mathrm{MeV}$ based on $\left\langle\frac{d E}{d x}\right\rangle$ for muons in polyvinyltoluene [3]. Green table entries indicate situations that result in only one strip with charge, while those in blue indicate situations in which both strips have some charge. As with the simulated multiplicity data in Figures 8 and 9, greater angles are more likely to yield two strips hit; this also makes sense geometrically.

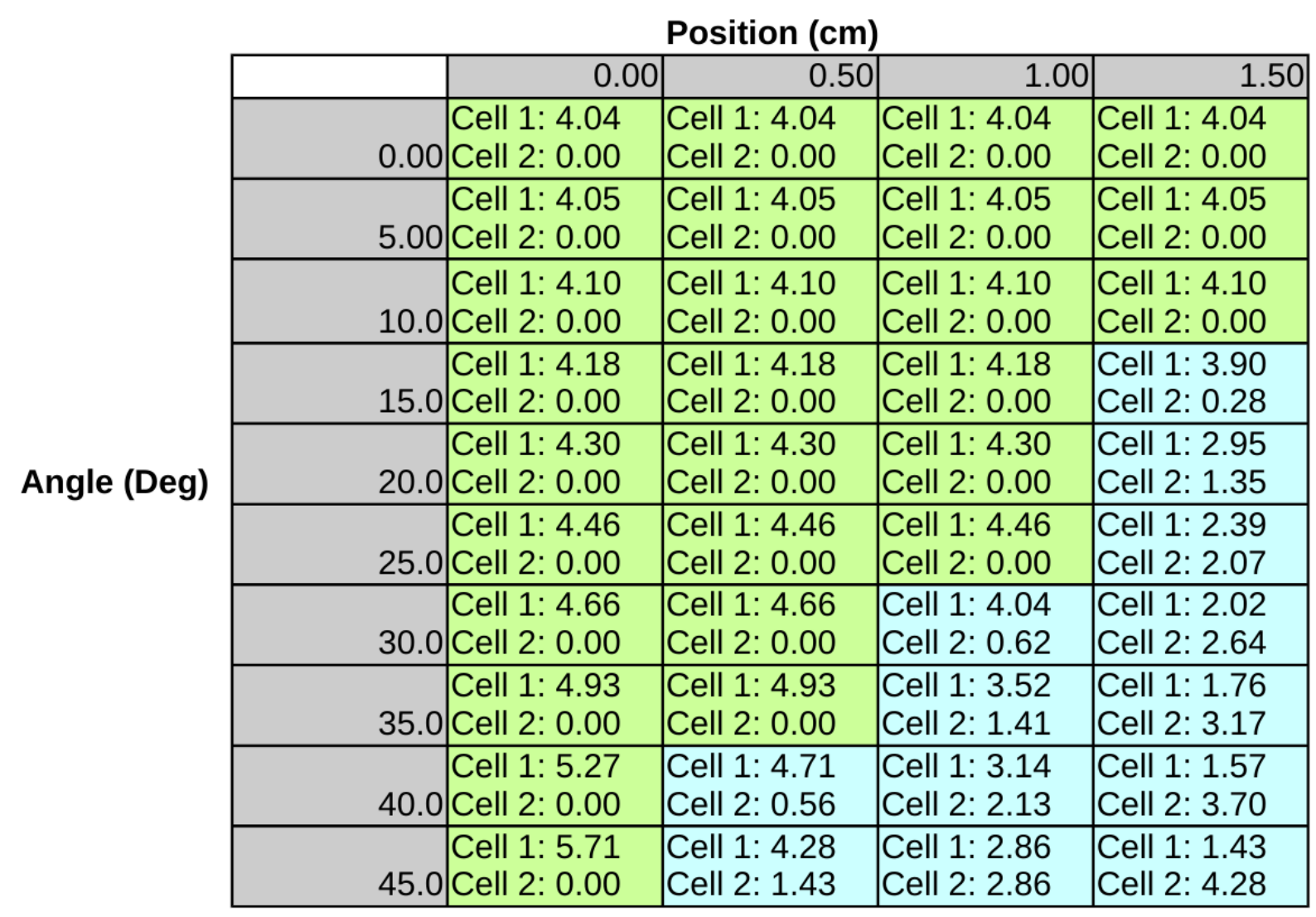

Figure 10: A map of expected energy deposition (MeV) in two adjacent rectangular scintillator strips as angle and position vary. Here, "angle" refers to the amount by which the incoming muon differs from normal incidence; "position" refers to the distance between its initial hit position and the edge of the first strip. Cases that yield only one strip with charge are shown as green table entries, and those that yield two are shown in blue. 


\section{Positional Back-projection and Comparison with Con- crete Scattering}

One of the toy model's primary goals is to determine the strip size needed for optimum detector resolution. A detector with sufficient resolution should result in an uncertainty comparable to or somewhat smaller than any multiple scattering effects from the pyramid; however, a detector with too-fine resolution results in an unnecessarily high channel count and therefore unnecessary cost. So, it is of interest to calculate the particles' hit positions at the detector planes, back-project the resulting trajectory to its starting place (or any other point desired), and compare the uncertainty inherent in this process to that arising from scattering.

As discussed in section 5, these studies used only those events that survived the "singles cut," i.e., those that yielded exactly one hit per detector plane. We therefore took the hit position in a given plane to be the center of the strip with non-zero energy deposition. Each plane gave either a $\mathrm{u}$ or a $\mathrm{v}$ coordinate, and these were combined to give one point in space for each detector.

The RMS error associated with a rectangular strip of width A is given by

$$
\sigma=\frac{A}{\sqrt{12}}
$$

so, for the 2-cm strips used here, $\sigma=\frac{0.02 m}{\sqrt{12}}$ or c. $0.01 \mathrm{~m}$. The calculated positions and their uncertainties can be back-projected to any plane by simply inserting them into the equation of a line (see Figure 11):

$$
\frac{X-X_{A}}{X_{B}-X_{A}}=\frac{Y-Y_{A}}{Y_{B}-Y_{A}}=\frac{Z-Z_{A}}{Z_{B}-Z_{A}}
$$

where $\left(X_{A}, Y_{A}, Z_{A}\right)$ and $\left(X_{B}, Y_{B}, Z_{B}\right)$ are the global coordinates corresponding to the hit positions at detectors one and two, respectively, and $(X, Y, Z)$ are the coordinates of the back-projected point. The result is a "circle of confusion," determined by the radius $r_{Y}$ in Fig. 11, that represents the detector system's inherent uncertainty at the distance in question. The goal, then, is to find a strip width for which this radius does not differ substantially from the deviation caused by multiple scattering. 


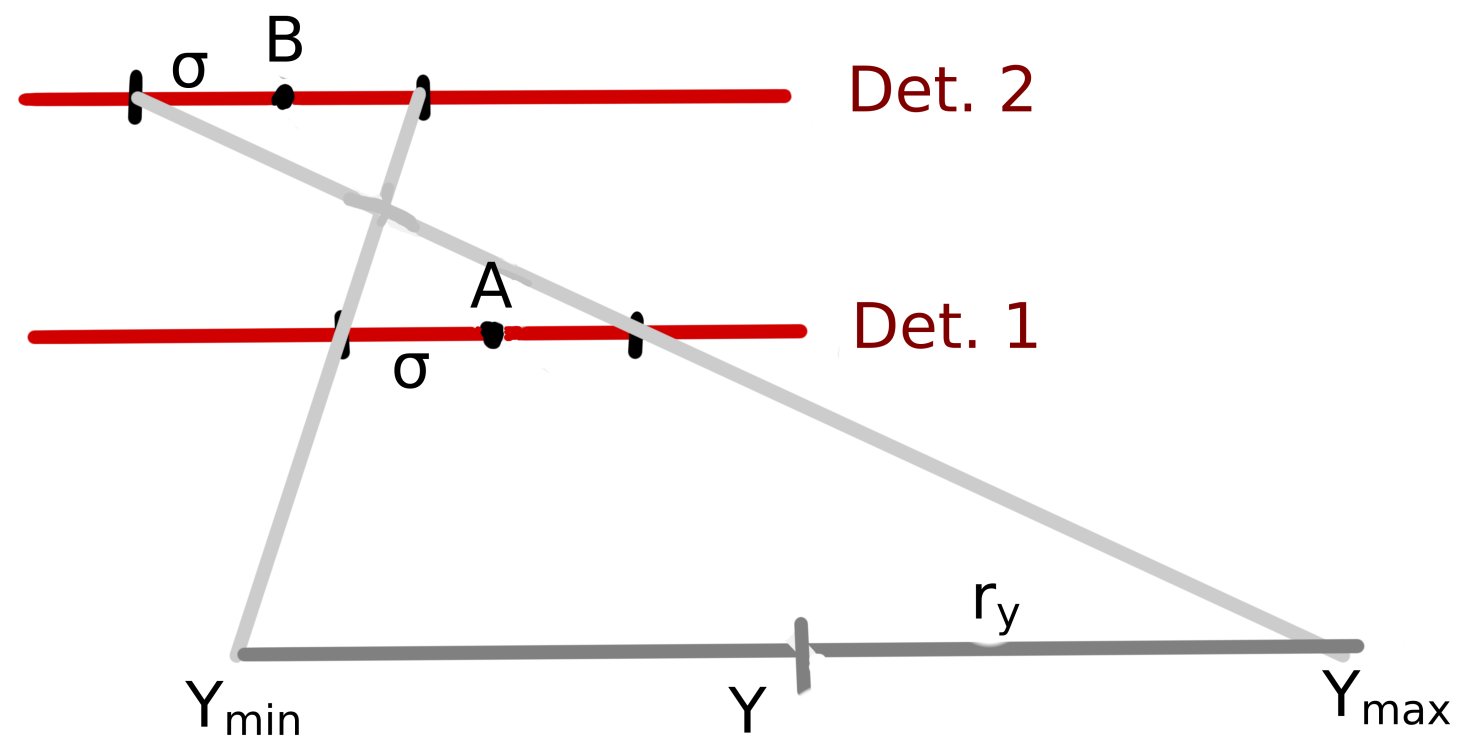

Figure 11: A 2D sketch of how the RMS "circle of confusion" radius is calculated. Since the strip width is the same in all planes, $\sigma$ is the same for detectors one and two.
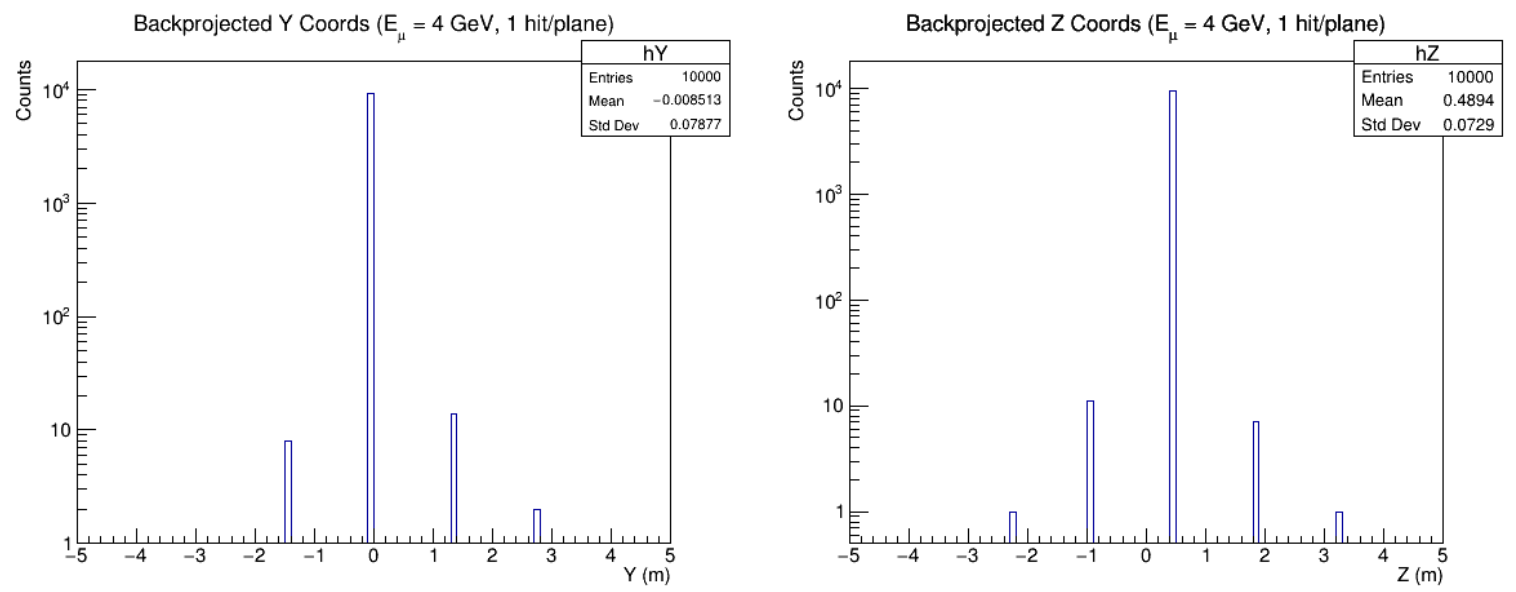

Figure 12: Preliminary results from the back-projection algorithm, using a run of 10,000 4GeV muons and no concrete. The only events used were those that produced one hit strip per detector plane; the beam was placed at normal incidence at the center of the target strips. Trajectories were then projected a distance of $140 \mathrm{~m}$, corresponding to the hypothetical center of the pyramid and the origin of the global coordinate system.

To validate the Root macro that back-projects these trajectories (without uncertainties), we began with a run of 10,000 4-GeV muons at normal incidence to the detectors, and this run did not include concrete. The calculated hit positions were back-projected $140 \mathrm{~m}$, which corresponds to a point $0.49 \mathrm{~m}$ above the - here hypothetical - center of the pyramid's base (see Figs. 3, 4). Two one-dimensional histograms containing the resulting Y and $\mathrm{Z}$ coordinates are given in Figure 12. In both dimensions, the majority of events yielded correct starting positions $(\mathrm{Y}=-0.01 \mathrm{~m}$ and $\mathrm{Z}=0.49 \mathrm{~m}$ respectively). Both distributions 
also have a very few values in non-central bins. The discrete locations at which these values occur, combined with the fact that they vanish for higher values of $\mathrm{E}_{\mu}$, indicate that they arise from muons that scatter to strips adjacent the target. So, the algorithm successfully back-projects trajectories as desired.

\subsection{Example Study: Void 25 m from Pyramid Surface}

Here we consider an example scenario in which there is a void of interest $25 \mathrm{~m}$ from the pyramid's surface, and we compare the back-projection uncertainty arising from multiple scattering to the RMS error inherent in the scintillator strips.

For the purposes of this simulation, our scenario can be modeled by a $25-\mathrm{m}$ long concrete block placed $25 \mathrm{~m}$ from the first plane of detector one. For simplicity, the particles exiting the pyramid were represented by a normal beam of 10,000 50-GeV muons placed just upstream of the concrete (see Fig. 7, for example). These trajectories were then backprojected from their calculated hit positions to the far end of the concrete, a total of $50 \mathrm{~m}$ from detector one. Figure 13 shows the results of this projection.
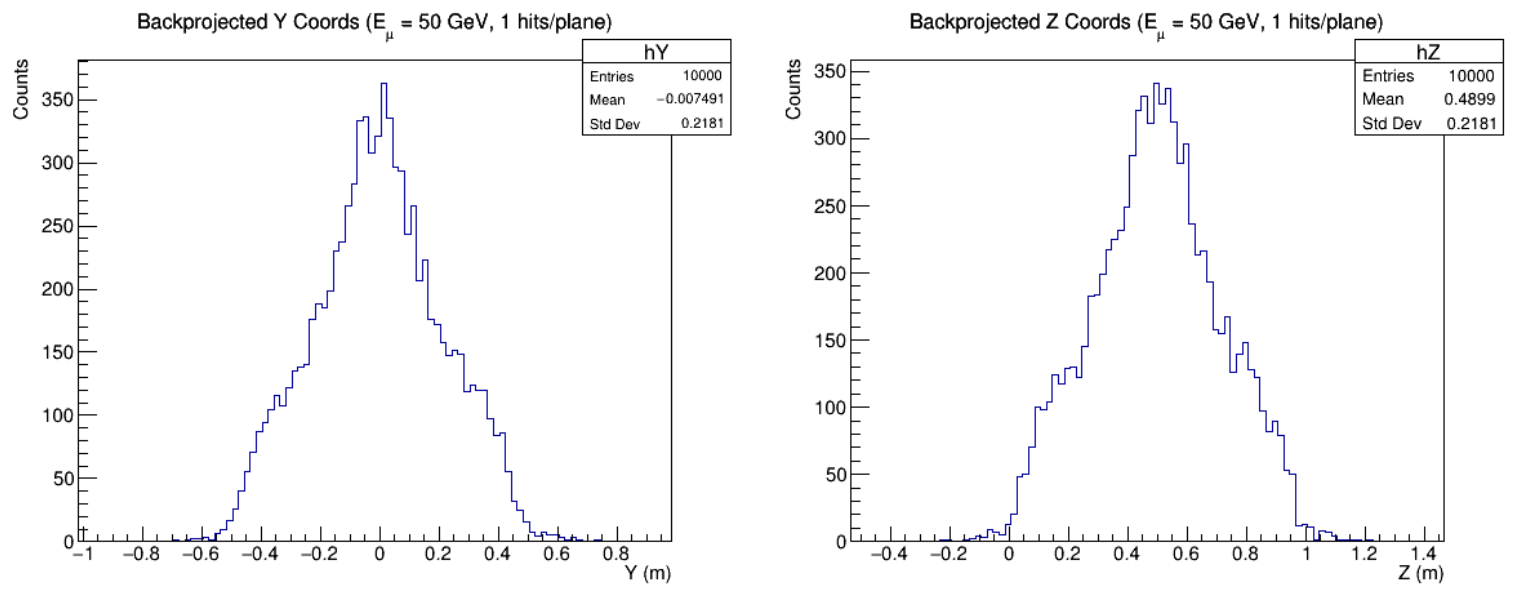

Figure 13: Results from the back-projection algorithm for a run including a 25-m block of concrete. The $10,00050-\mathrm{GeV}$ muons from this run were back-projected to the far end of the block - i.e., $50 \mathrm{~m}$ from detector 1 and $89 \mathrm{~m}$ from the hypothetical pyramid center. The resulting distribution is a reflection of multiple-scattering effects in the concrete; comparing the standard deviations of this and similar results to the RMS error of the scintillator strips provides insight on the strip size needed for sufficient position resolution.

Both plots are again centered on the beam's true starting point (as before, $\mathrm{Y}=-0.01$ $\mathrm{m}$ and $\mathrm{Z}=0.49 \mathrm{~m}$ ). The continuous distributions in this case, however, directly reflect the effects of multiple scattering inside the concrete.

Using the calculation method outlined in Figure 11, the RMS circle of confusion radius coming solely from the detector resolution is approximately $\mathrm{r}=0.29 \mathrm{~m}$. This is slightly larger than, but quite similar to, the standard deviation of about $0.22 \mathrm{~m}$ in both plots above. In this highly specific scenario, then, a 2-cm rectangular scintillator design would likely have sufficient resolution. However, a thinner rectangle or a triangular design might still provide advantages, as we do not want the inherent detector resolution to affect our capability of detecting various unknowns in the pyramid. 


\section{$7 \quad$ Use of $\frac{d E}{d x}$ in Determining Muon Momentum}

It is possible that the charge collected by the detector cells can be used to sort muons by their incident momenta. If successful, this approach could yield clearer image reconstruction by allowing us to use only those muons in an ideal energy range - eliminating too-soft particles that experience significant scattering and too-hard particles that may not be sensitive to variations in the material of the pyramid. Accordingly, we explored the correlations between muon momentum and energy deposited in the scintillator strips, and this is outlined below. Concrete was omitted in all of these runs; $\mathrm{E}_{\mu}$ here refers to the muon's energy before it reached the first detector plane.

Figure 14 depicts example results from two metrics for measuring energy absorption in the scintillator; on the left, muon hit multiplicity across all four detector planes, and on the right, total energy deposited across all planes for the same run. As expected, the latter is a Poisson distribution. It should be noted that the hit-multiplicity plot is shown on a log scale; it appears that a total of 4 hits per event - and therefore 1 hit per plane - is most common at this energy $\left(\mathrm{E}_{\mu}=40 \mathrm{GeV}\right)$. This was discussed more thoroughly in section 5 .
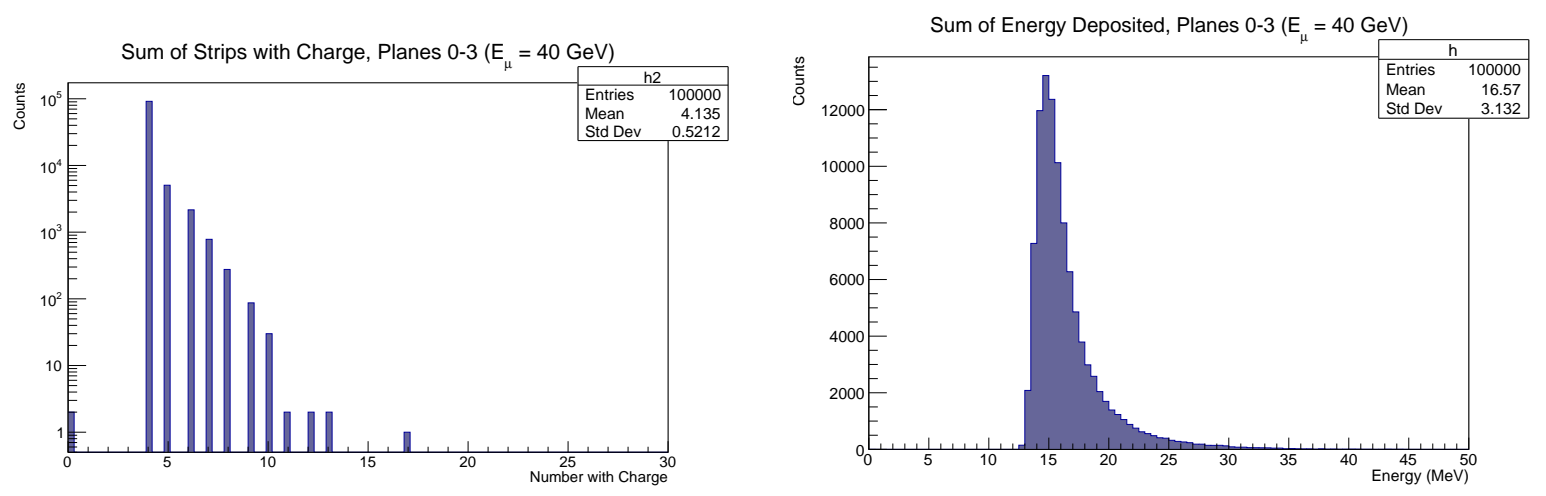

Figure 14: Left: distribution of total number of strips hit in all four detector planes after a run of 100,000 muons, with $\mathrm{E}_{\mu}=40 \mathrm{GeV}$. Right: distribution of total energy deposited in all four planes after the same run.

The left side of Figure 15 shows the mean total energy deposited in all four detector planes as a function of increasing incident muon energy; the right side of the figure shows the mean difference between incident and final muon energies as a function of the same. The latter plot demonstrates a noticeable increase in energy loss as $\mathrm{E}_{\mu}$ increases. The former, on the other hand, shows very little absolute change over the range of momenta tested.

The energy collected by the detectors, then, does not vary measurably with the energy of the incident particle. Although the muons' overall energy loss is strongly correlated with their initial momenta, only a fraction of this lost energy is absorbed by the detectors. The remainder is lost to the surroundings, primarily by means of gamma rays, which unlike electrons do not strongly interact with the scintillator. It is worth noting that this effect is partially due to the small size of the detectors in the simulation; a larger array of detectors, like that filling an entire shipping container, would result in improved energy collection. However, this would likely not be enough to account for the discrepancy in Fig. 15. So, before $\frac{d E}{d x}$ can be used to tag muons effectively, a way of increasing deposition in the detector planes is needed. 

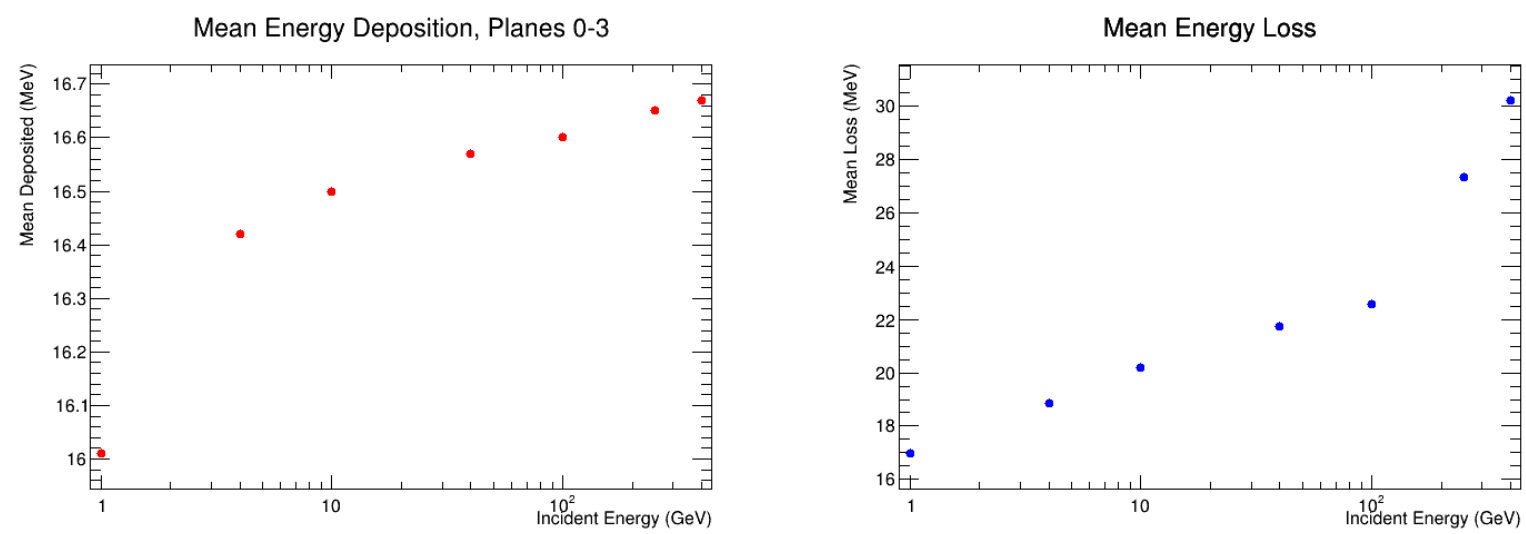

Figure 15: Left: the mean total energy deposited in all four scintillator planes as a function of incident muon energy. Right: the mean total energy lost by the muon as a function of incident energy. Particularly for muons at higher energies, there is a large discrepancy between this total energy loss and the energy deposited in the detectors themselves.

\subsection{Basic Detector Design vs. Additional Pb Layer}

To improve the correlation between incident muon momentum and energy deposition in the scintillators, we inserted a sheet of lead between detectors one and two (see Figure 16). The width and height of this sheet matched that of the detector planes; its thickness varied between $1 \mathrm{~cm}$ and $2 \mathrm{~cm}$, while its exact placement ranged from $12 \mathrm{~cm}-20 \mathrm{~cm}$ upstream of detector two. This addition was intended to result in a greater number of showers from secondary gamma rays and therefore an increased number of strips hit in the second detector. However, it had the potential disadvantage of degrading the detectors' position resolution.

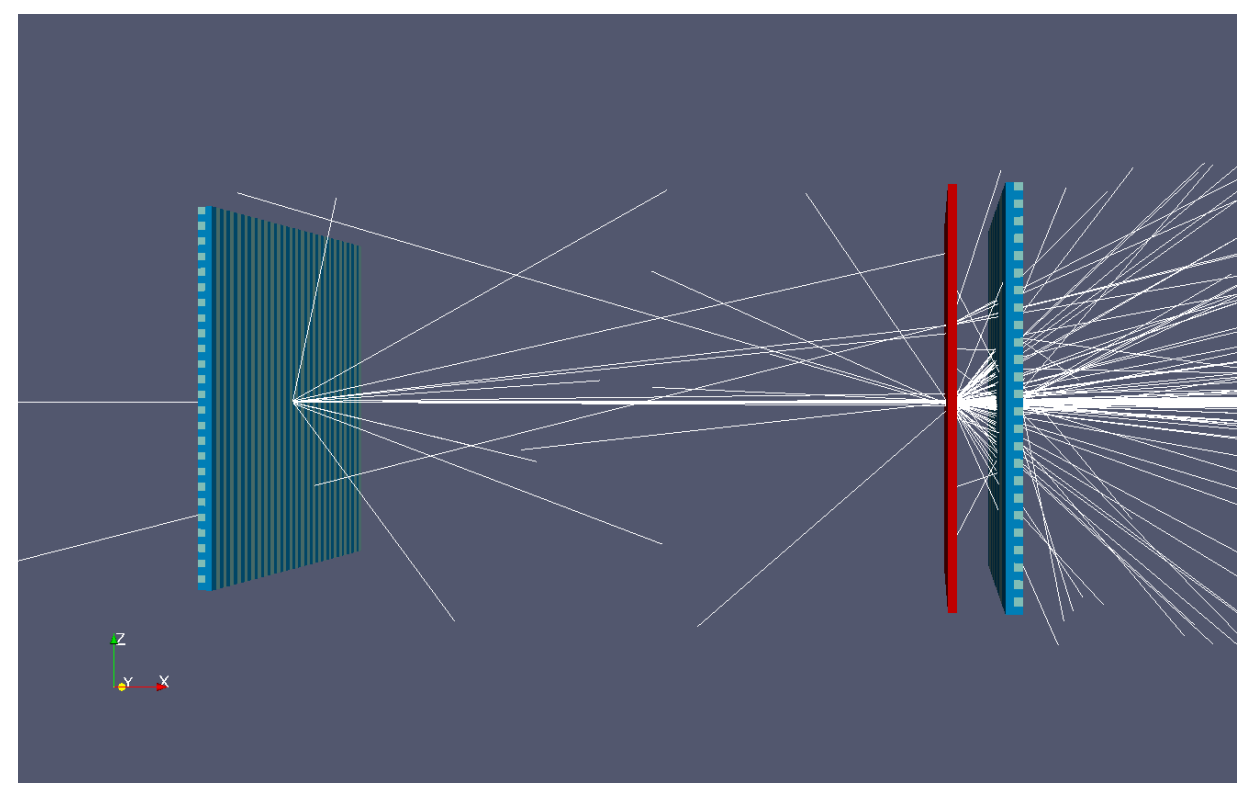

Figure 16: The modified detector design includes a lead sheet of varying thickness between the two detectors, and the placement of this sheet ranged from $12 \mathrm{~cm}-20 \mathrm{~cm}$ upstream of detector 2. The goal of this addition was to increase the number of secondaries striking detector 2 without substantially degrading position resolution. 
As shown on the left side of Figure 17, adding lead did have the intended effect of increasing hit multiplicity for higher-energy particles. The red data points represent the mean number of strips hit in detector two only for the original detector design (no $\mathrm{Pb}$ ); the green and blue data represent the mean strips hit for configurations with $1 \mathrm{~cm}$ and $2 \mathrm{~cm}$ of lead, respectively. The lead sheets for both cases were placed $12 \mathrm{~cm}$ away from the detector. The correlation between $\mathrm{E}_{\mu}$ and mean hit multiplicity is strongest for the 2-cm lead scenario.

However, these results also indicate that the modified detector design is likely unusable. The absolute increase in multiplicity over the range of energies tested is small (with a mean of c. 2.2 strips at $\mathrm{E}_{\mu}=1 \mathrm{GeV}$ and c. 3.2 strips at $\mathrm{E}_{\mu}=400 \mathrm{GeV}$ ), and this range is itself larger than the probable real-life range of interest. Furthermore, the fraction of events that resulted in a substantially greater multiplicity is quite small. The plot on the right side of Fig. 17 shows the percentage of events, out of a 10,000-event run, that yielded more than ten strips hit in detector two as a function of increasing $\mathrm{E}_{\mu}$. Here, the blue points correspond to the 1-cm $\mathrm{Pb}$ configuration, while the red points correspond to $2 \mathrm{~cm} \mathrm{~Pb}$. Even at the highest energy simulated $\left(\mathrm{E}_{\mu}=400 \mathrm{GeV}\right)$, the fraction of events satisfying this condition does not exceed c. 3.5\%. In short, adding a lead sheet between the two detectors is not a practical means of improving hit multiplicity for tagging incident $\mathrm{E}_{\mu}$. This design was not used in any of the other studies described in this paper.
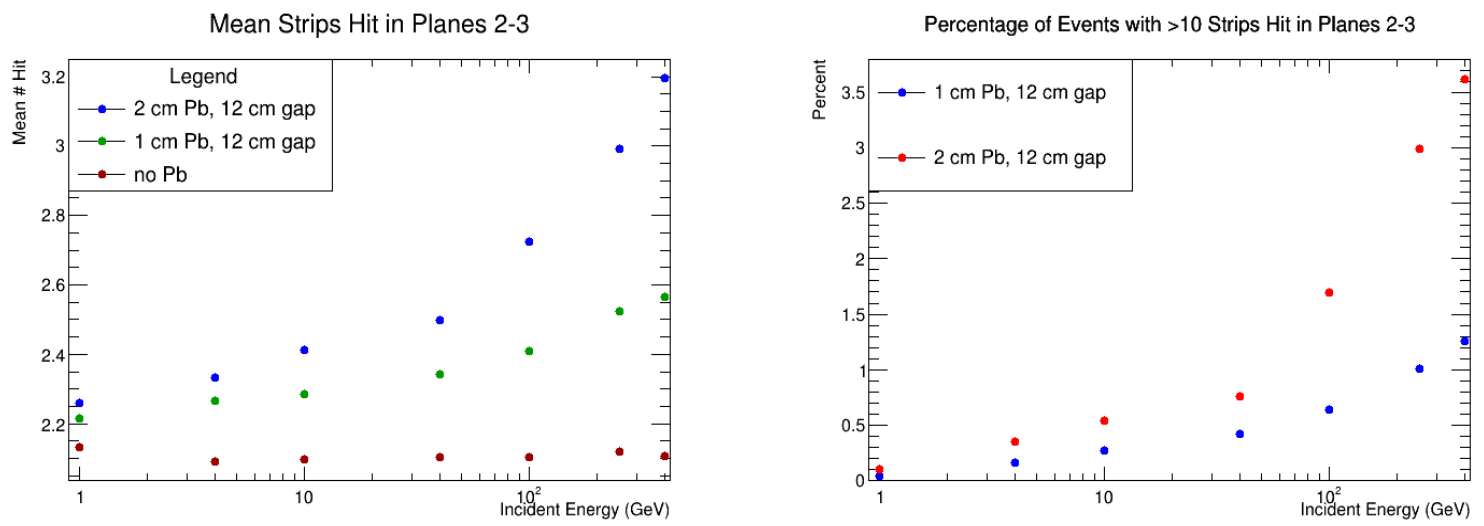

Figure 17: Left: the mean number of strips hit in detector 2 as a function of incident muon energy, for various lead configurations; 10,000 muons per run. Right: out of 10,000 muons per run, the percentage of events for which more than 10 strips were hit in detector 2 . Adding $\mathrm{Pb}$ yielded a strong correlation between incident muon energy and number of strips with charge, as hoped. However, this configuration is likely unusable.

\section{Summary}

Here, we have considered the use of rectangular scintillator strips in the EGP mission's muon telescopes. Although the design discussed does provide sufficient position resolution in specific test cases, there is much room for improvement. For example, replacing the rectangular scintillators with triangular ones may further improve positional and angular resolution and even turn the presence of multiple hits into an advantage. In addition, inserting a lead sheet between the detectors is not a feasible means of improving the correlation between incident muon momentum and energy deposition in the scintillators; therefore, in order to tag particles by their incoming energies, another approach will be needed. 


\section{Acknowledgments}

The author sincerely thanks Alan Bross and Henry Frisch for their guidance, support, and mentorship on all aspects of the project and Ralf Ehrlich for his expert advice regarding GEANT4. I thank Anna Pla-Dalmau, Edmond C Dukes, Sophie Dukes, Adam Para, Patrick La Riviere, Omar Shohoud, Christian Sodano, and Leah Welty-Rieger for all their feedback and guidance. My thanks also to the SIST committee for making this summer research possible. This manuscript has been authored by Fermi Research Alliance, LLC under Contract No. DE-AC02-07CH11359 with the U.S. Department of Energy, Office of Science, Office of High Energy Physics.

\section{References}

[1] L. Alvarez, A. Jared, F. Anderson, F. Bedwei et al., Search for Hidden Chambers in the Pyramids, Science 167 (1970) 832-839.

[2] K. Morishima et al., Discovery of a big void in Khufu's Pyramid by observation of cosmic-ray muons, Nature 552 (2017) 386-390.

[3] C. Patrignani et al (Particle Data Group). Chinese Physics C, 2016 40(10) (2016) 100001.

[4] GEANT4 Collaboration, V. Ivanchenko, Geant4 toolkit for simulation of HEP experiments, Nuclear Instruments and Methods in Physics Research A 502 (2003) 666-668.

[5] "GEANT4 Tool Kit.” http://geant4.cern.ch/.

[6] "ROOT." https://root.cern.ch/.

[7] Rene Brun and Fons Rademakers, ROOT - An Object Oriented Data Analysis Framework, Proceedings AIHENP'96 Workshop, Lausanne, Sep. 1996, Nucl. Inst. and Meth. in Phys. Res. A 389 (1997) 81-86.

[8] Ahrens, James, Geveci, Berk, Law, Charles, ParaView: An End-User Tool for Large Data Visualization, Visualization Handbook, Elsevier, 2005, ISBN-13: 978-0123875822 